\title{
Use of fiber optic technology for relative humidity monitoring in RPC detectors
}

\author{
M. A. Caponero ${ }^{1}$ \\ Centro Ricerche ENEA Frascati \\ via E. Fermi 45, 00044 Frascati, Rome, Italy \\ E-mail: michele.caponero@enea.it \\ and \\ Istituto Nazionale Fisica Nucleare, Laboratori Nazionali Frascati \\ via E. Fermi 40, 00044 Frascati, Rome, Italy
}

\section{A. Polimadei}

Centro Ricerche ENEA Frascati

via E. Fermi 45, 00044 Frascati, Rome, Italy

\section{Benussi, S. Bianco, S. Colafranceschi, L. Passamonti, D. Piccolo, D. Pierluigi,}

A. Russo

Istituto Nazionale Fisica Nucleare, Laboratori Nazionali Frascati

via E. Fermi 40, 00044 Frascati, Rome, Italy

\section{Ferrini, F. Felli, G. Saviano, C. Vendittozzi}

Dept. ICMA, University "La Sapienza"

via Eudossiana 18, 00184 Rome, Italy

and

Istituto Nazionale Fisica Nucleare, Laboratori Nazionali Frascati via E. Fermi 40, 00044 Frascati, Rome, Italy

Best performance of Resistive Plate Counter detectors calls for accurate control and monitoring of gas parameters within the full volume of the detector. A novel application is proposed for Relative Humidity (RH) monitoring of gas in RPC detectors by means of Fiber Bragg Grating (FBG) optical sensors, and preliminary results are reported.

XI workshop on Resistive Plate Chambers and Related Detectors (RPC2012)

INFN-Laboratori Nazionali di Frascati, Italy

February 5-10, 2012

\footnotetext{
${ }^{1}$ Speaker
} 


\section{Introduction}

Environmental parameters such as temperature, atmospheric pressure, etc strongly affect the working point of all nuclear and subnuclear physics detectors based on the ionization of a gas medium. For RPC in particular, RH affects both the resistivity of bakelite and the response of gas, thus resulting in an effect on the working point. Effect of environmental parameters on the RPC working point has been studied in the past [1, 2, 3].

Since RH is one of the parameters that can effectively affect the efficiency and the working point of individual detector chambers, distributed real-time sensing of such parameter is an important issue. In this paper we propose the use of optical sensing by Fiber Bragg Grating technology to develop a distributed RH monitoring system. The proposed technology offers the valuable advantage of immunity to electromagnetic disturbances and reduced wiring thanks to the possibility of cabling numerous sensors in series on one optical fibre.

\section{Fiber Bragg Grating sensors}

FBG (Fiber Bragg Grating) sensors are made by means of the writing of a diffraction grating in the core of an optical fibre; writing of grating is obtained by exposing the core to UV structured light, thus producing a modulation of its refractive index $[4,5]$. The grating usually is about $5 \mathrm{~mm}$ long; it is the only sensing segment of the optical fiber, the rest of the fiber being used to connect the sensor to the opto-electronic analysis system. If broad-band light is launched in the fibre, it is diffracted at the grating and a narrow-band light signal is back-reflected along the fiber. The peak wavelength value of the signal is determined by the value of both the pitch of the grating and of the effective refractive index at the grating. If the FBG is tensioned and/or heated, changes of the value of the pitch and/or the value of the effective refractive index occurs, thus causing a shift of the peak wavelength value of the signal. Thus, an FBG sensor can monitor temperature/strain of a structure to which it has been put in thermal/mechanical contact [6]. The basic equation governing the signal of an FBG sensor can be written as

$$
\Delta \lambda=a \cdot \Delta T+b \cdot \varepsilon
$$

being: $\Delta \lambda$ the peak wavelength shift; $\Delta T$ the temperature variation; $\varepsilon$ the strain; $a$ and $b$ constants.

Thanks to the spectroscopic nature of the FBG technology, sensors are immune to electromagnetic disturbances and can be operated in WDM (Wavelength Division Multiplexing); thus they do not suffer high magnetic fields and many sensors can be connected in-series on one optical fibre, thus reducing the amount of wiring required for installing a large number of sensors. Moreover FBG sensors have already been proved to have radiation hardness feature that make them suitable for HEP experiments and they have already been proposed for metrological monitoring in HEP detectors [7, 8]. 


\section{Experimental Method}

We propose to use an FBG sensor inserted in the gas conduit upstream each chamber of the RPC detector, thus monitoring the distribution of the RH value over the full detector. The advantage of the proposed technology lies in its insensitivity to electromagnetic interferences and in its reduced wiring requirements. Both features come from the spectroscopic character of the technology and make it of great interest for applications related to High Energy Physics detectors, which usually have very large volume and are operated in high magnetic field. Such features become of particular interest for RPC detectors being composed of a large number of individual chambers and being operated at high voltage. The large number of individual chambers calls for a large number of sensors to be deployed, thus finding an effective advantage in the possibility to adopt in-series interconnection of tens of sensors along one optical fibre.

Standard commercial FBG sensors are produced on standard telecommunication single mode optical fibre with acrylate coating. To make the FBG sensor, the acrylate coating is removed at the selected location and after the inscription of the diffraction grating by UV radiation exposition, a new coating is put in place. Standard FBG sensor have either acrylate or polyimide recoating, acrylate being the usually adopted for temperature sensing, polyimide being usually adopted for structural sensing. Polyimide recoating is usually adopted for structural sensing because of its better mechanical properties that allow to obtain excellent structural bonding with the component to be monitored. Since polyimide also exhibit peculiar swelling property, FBG sensors with polyimide recoating can be expected to allow RH monitoring. In fact, swelling of the polyimide recoating turns out in tensioning of the coated Bragg grating with subsequent encoding of the $\mathrm{RH}$ variation in the Bragg wavelength shift. Such effect is of no concern in structural monitoring since the glue used for structural bonding shields the FBG sensor by the environment humidity. Use of special recoating for production of RH sensors by FBG technology have been proposed, but we here propose the use of standard commercial polyimide recoated FBG sensors to develop a densely distributed RH monitoring system of gas fluxed in RPC detectors.

A prototype of the proposed sensor was realised by a standard commercial polyimide recoated FBG sensors made by Welltech Instrument Company ltd, mod. bare/1550/polyimide. The sensor was inserted in a sealed box provided with a reference RH and temperature sensors; the box was also provided with inlet and outlet pipe joints at opposite sides to flux gas through the box. Fig.1 shows a schematic drawing of the layout; Fig.2 shows a picture of the inside of the box. The adopted RH and temperature monitoring system is mod. Minipc70 made by Mitchell Company. The temperature reference sensor was adopted in order to perform the required temperature compensation, being the FBG sensor sensitive to temperature according to the basic equation governing the FBG sensing technology. Special attention was paid to the arranging of the FBG sensor inside the box in stable mechanical configuration in order to avoid uncontrolled settlement during usage, as that would result in wavelength shift inconsistent with both temperature and $\mathrm{RH}$.

\section{Experimental Results}

Measurements were done in the RPC Test Facility of the ASTRA laboratory at Frascati National Laboratory of INFN. Measurements were conducted using a RH calibration kit with 
standards for $10 \% \mathrm{RH}, 25 \% \mathrm{RH}, 65 \% \mathrm{RH}$. Standards where inserted in the box in sequence from the lowest value to the largest value and then back to the lowest value. The standard with the largest value was left in the box for longer time than the other standards, leaving the box non perfectly sealed in order to have a slight variation of the RH value. Results are shown in Fig.3. The plot shows RH, temperature and FBG signal vs time. Temperature is scaled in order to be fitted in the plot, to allow evidence of the dependence of the FBG signal on temperature. To work out RH value from FBG signal, temperature compensation must be applied according to equation

$$
\Delta R H=\alpha T+\beta \Delta \lambda
$$

Values of parameters $\alpha$ and $\beta$ are found by fitting of data shown in Fig.3, getting the following result

$$
\begin{aligned}
& \alpha=-0.189{ }^{\circ} \mathrm{C}^{-1} \\
& \beta=20.35 \mathrm{~nm}^{-1}
\end{aligned}
$$

Result of equation

$$
\Delta R H=-0.189 \cdot T+20.35 \cdot \Delta \lambda
$$

is shown in Fig.4, where RH values measured by reference sensor are shown too.

Fig.4 shows a good agreement between values of RH measured by the reference sensor and the FBG sensor, a part from an evident discrepancy at the beginning of the measurements (time $<2 \mathrm{~h}$ ). Such discrepancy could be due to hysteresis of the swelling of the polyimide, suggesting to adopt for future measurement a preliminary cycling of the sensor. We propose to use an FBG sensor inserted in the gas conduit upstream each chamber of the RPC detector, thus monitoring the distribution of the $\mathrm{RH}$ value over the full detector. The advantage of the proposed technology lies in its insensitivity to electromagnetic interferences and in its reduced wiring requirements. Both features come from the spectroscopic character of the technology and make it of great interest for applications related to High Energy Physics detectors.

\section{Conclusions and future work}

We have proposed the use of a distributed FBG sensor system to monitor the distribution of the RH value over large RPC detectors. The proposed monitoring system is based on deploying an FBG sensor upstream each individual chamber, in the gas conduit of the RPC detector. Preliminary experimental measurements have been worked out to develop a prototype sensor. Results show that improvements are required in order to succeed in increasing both precision and repetitiveness. Long term measurements are planned with preliminary cycling of the sensor to overcome hysteresis effects. Future measurement will be performed with larger number of $\mathrm{RH}$ standard values and with continuous $\mathrm{RH}$ variation of gas fluxed through controlled humidifier.

\section{References}

[1] L. Benussi et al., Sensitivity and environmental response of the CMS RPC gas gain monitoring system, JINST 4: P08006,2009; e-Print: arXiv:0812.1710 [physics.ins-det].

[2] L. Benussi et al., A New approach in modeling the response of RPC detectors, Nucl.Instrum.Meth.A661:S182-S185,2012; e-Print: arXiv:1012.5508 [physics.ins-det]. 
[3] S. Colafranceschi et al., Operational experience of the gas gain monitoring system of the CMS RPC muon detectors, Nucl.Instrum.Meth.A617:146-147,2010.

[4] K.O. Hill, G. Meltz, Fiber Bragg grating technology fundamentals and overview, Journal of Lightwave Technology 15 (1997).

[5] I. Bennion, J.A.R. Williams, L. Zhang, K. Sugden, N.J. Doran, UV written in-fibre Bragg gratings, Optical and Quantum Electronics 28 (1996).

[6] Y.J. Rao, In-fibre Bragg gratings, Measurement Science and Technology 8 (1997).

[7] E. Basile et al., Micrometric position monitoring using fiber Bragg grating sensors in silicon detectors,in proceedings of the 9th ICATPP Conference on Astroparticle, Particle, Space Physics, Detectors and Medical Physics Applications, Villa Erba, Como, Italy, 1721 Oct 2005; e-Print: physics/0512255.

[8] L. Benussi et al., The Omega-like: A novel device using FBG sensors to position vertex detectors with micrometric precision, Nucl.Phys.Proc.Suppl.172:263-265,2007.

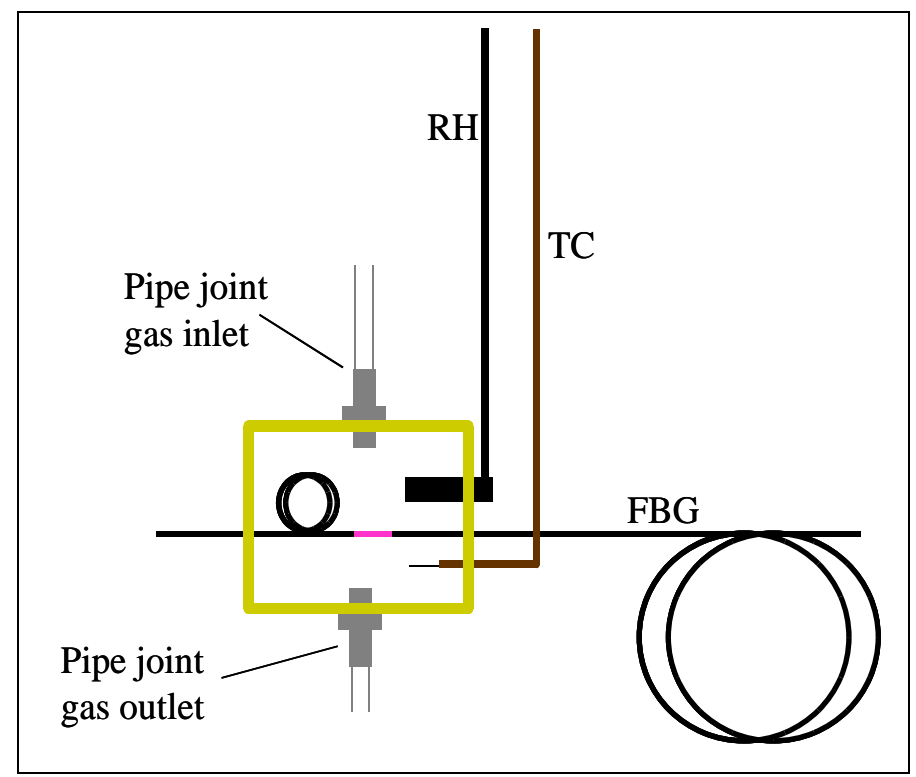

Fig.1. Schematic drawing of the experimental set-up. TC: thermocouple for reference temperature monitoring; RH: capacitive sensor for RH reference monitoring; FBG: optical fibre and FBG sensor. 


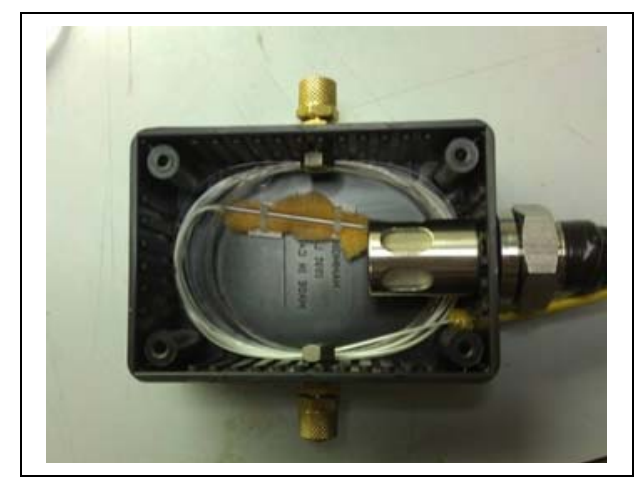

Fig.2. FBG sensor arranged in the box with $\mathrm{T}$ and $\mathrm{RH}$ reference monitoring sensors.

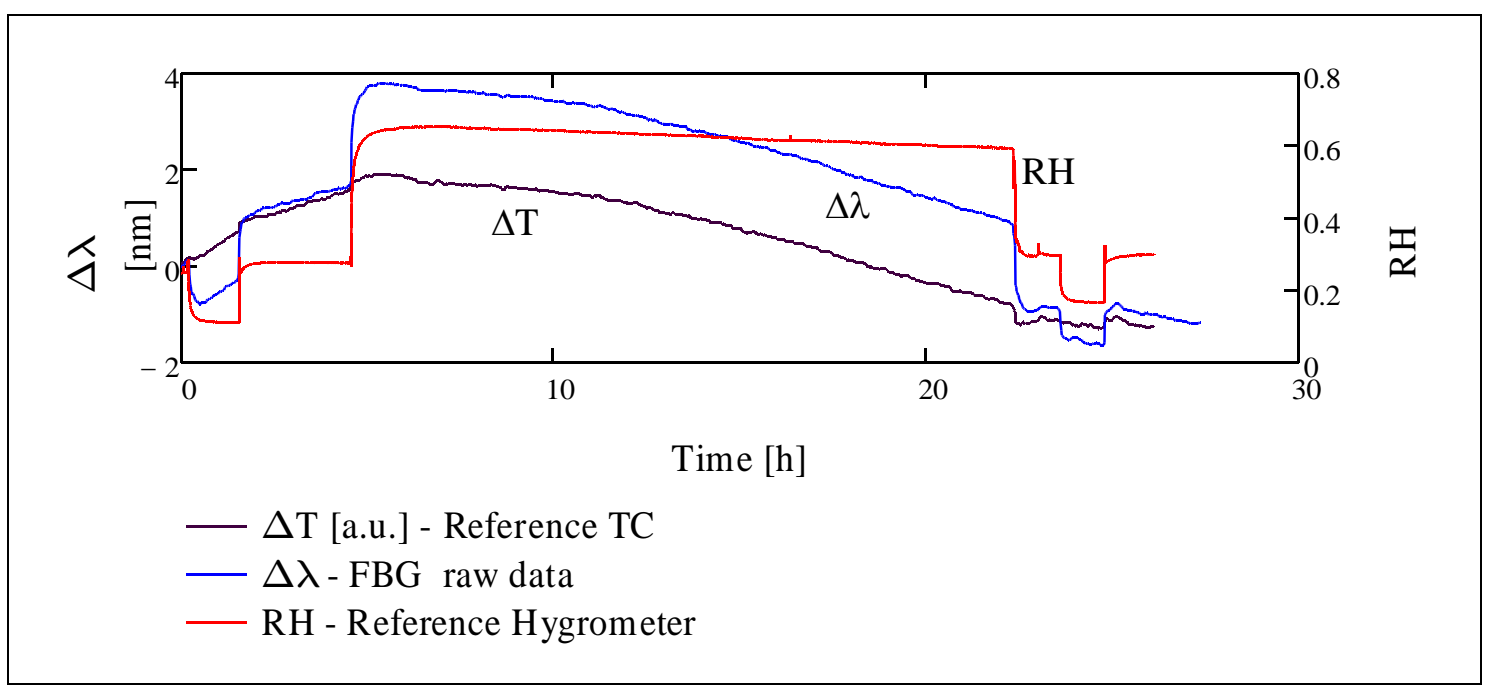

Fig.3. Plot of experimental data. Left Y-axis: FBG Bragg wavelength shift. Right Y-axis: relative humidity. $\Delta \mathrm{T}$ data are reported in arbitrary units to fit plot and allow qualitative comparison with FBG data.

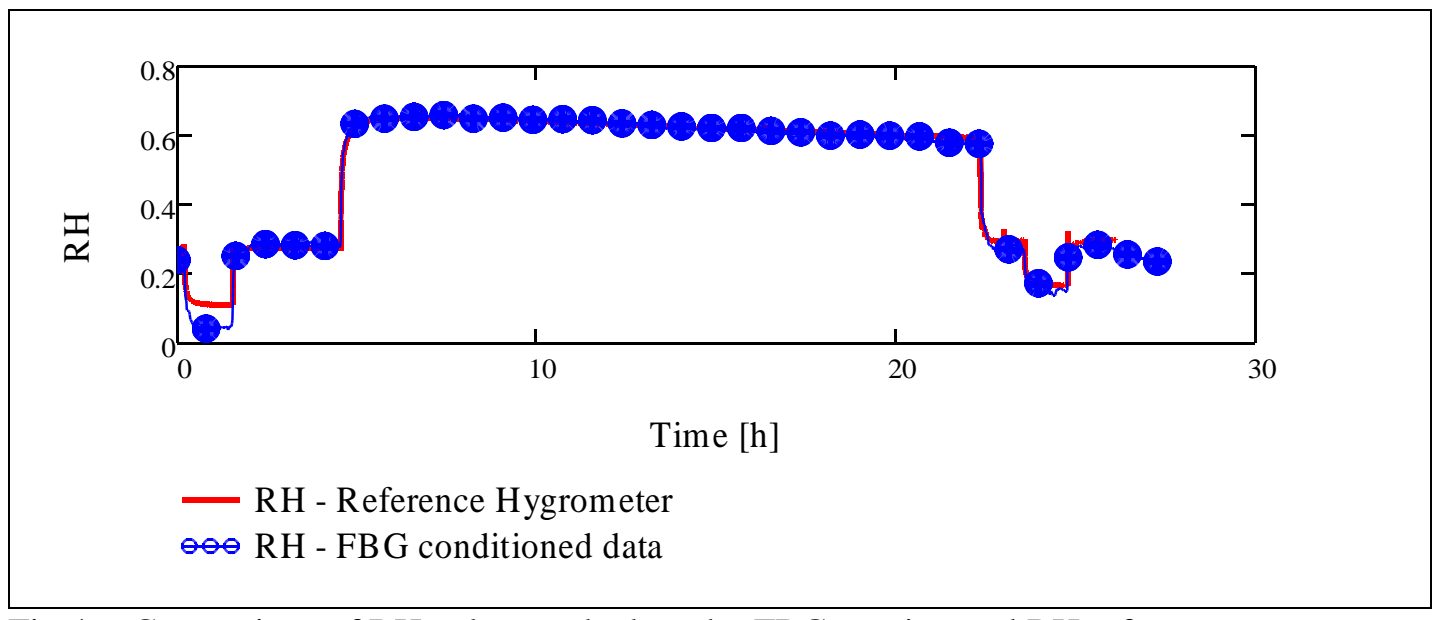

Fig.4. Comparison of RH value worked out by FBG sensing and RH reference measurement. 